\title{
Lutheal phase stimulation and fertility and first outcomes.
}

\author{
Luis M Campos Olmedo ${ }^{1 *}$, Miguel de J López Rioja ${ }^{2}$, Cristina M Sánchez González ${ }^{3}$ Paula N Zavala González \\ Yesenia Recio López ${ }^{5}$,Alejandro Chavez Badiola ${ }^{6}$
}

${ }^{1}$ Medical Coordinator, New Hope Fertility Center Mexico city, Mexico

${ }^{2}$ Medical Director, New Hope Fertility Center Guadalajara, Mexico

${ }^{3}$ Medical Associate, New Hope Fertility Center Mexico city, Mexico

${ }^{4}$ Embriology coordinator, New Hope Fertility Center Mexico city, Mexico

${ }^{5}$ Medical Associate, New Hope Fertility Center Mexico city, Mexico

${ }^{6}$ General Director, New Hope Fertility Center Mexico, Mexico

\begin{abstract}
During the menstrual cycle, ovarian follicles show dynamic morphologic and endocrine changes. Traditionally, folliculogenesis has been described to begin at the luteal phase with follicular recruitment, afterwards during the early follicular phase; a dominant follicle is selected while the rest undergo atresia. However, recent studies suggest that small antral follicles remain during the luteal phase, which do not undergo atresia and thus, are still capable of ovulating and can even result in pregnancy. These findings offer new possibilities for ovarian stimulation in selected cases.

Ovarian stimulation not started at the early follicular phase, (unconventional stimulation), may offer advantages against traditional protocols, such as flexibility to initiate stimulation at any given moment during the menstrual cycle. Suitable for oncology patients aiming to preserve fertility and a higher yield of oocytes retrieved per started cycle, which could be particularly attractive in patients with an initial poor ovarian response.

The efficacy of the luteal phase stimulation protocols is promising compared to single-stimulation protocols. To our knowledge, we report the first series of cases of unconventional stimulation (luteal phase) in Mexico and Latin America.
\end{abstract}

Keywords: Luteal phase protocols, Ovarian stimulation, Stimulation protocols.

\section{Introduction}

Traditional theory of folliculogenesis states that, during follicular phase, there is an antral follicular cohort from which a dominant follicle is selected, while the remaining follicles undergo atresia $[1,2]$. Studies conducted on animal reproduction have proven successful ovulation happening in both phases of the cycle (follicular and luteal phase), with pregnancy occurring with luteal phase ovulation in some mammal species [3]. Furthermore, recent evidence suggests that folliculogenesis in humans may also occur at two peaks (wave theory), supporting the concept of two or more cohorts of antral follicles during each cycle, where the development of a dominant follicle is preceded by anovulatory cohorts [4], capable of ovulating with appropriate luteinizing hormone (LH) stimulation resulting in pregnancy $[3,5]$. The latter becoming theory behind luteal phase stimulation (LPS) protocols.

Although LPS was traditionally considered as a last resort for cryopreservation in oncology patients [5-7]. Its current indications have broadened to patients with low ovarian response, cases of premature ovulation when aiming to shorten timing towards ovarian stimulation and for oncology patients [8]. Reported benefits of luteal phase stimulation can be summarized in shortened periods towards follicular stimulation, a higher number of retrieved oocytes per started cycle and as a means to avoid cycle cancellation following spontaneous ovulation [2].
When using LPS with the aim of maximizing oocyte yield per started cycle, it is suggested that double stimulation protocols should be considered, and could even become an alternative for patients with initial poor responses such as patients with polycystic ovarian syndrome (PCOS), or patients with poor ovarian reserve [4,9]. Several double stimulation protocols have been described in the recent literature with the most promising being:

\section{1) New York proposition}

Initial stimulation: Started with a minimal stimulation protocol as described elsewhere. In brief, ovarian stimulation begins on early follicular phase with a daily dose of $50 \mathrm{mg}$ clomiphene citrate (CC), followed by human Menopausal Gonadotropin (hMG) injections every other day (75 to $225 \mathrm{IU})$, until a leading follicle reaches $18 \mathrm{~mm}$. At this point follicular maturation is initiated with a gonadotrophin releasing hormone agonist (GnRH agonist) and oocyte retrieval (OR) performed $34 \mathrm{hrs}$ following injection.

LPS: Started two to seven days following OR, with a low dose gonadotropins regime of daily injections (75-150 IU/day) with the addition of either daily CC $(25-100 \mathrm{mg} /$ day $)$ or letrozole (2.5-5 mg/day). After 5 days of stimulation, ultrasound and blood hormone monitoring is resumed until a leading follicle reaches $18 \mathrm{~mm}$. At this point, trigger with either human chorionic 
gonadotrophin (hCG) (5000 IU) or GnRH agonist (0.1 mg). OR is performed 34 hours later [4] (Figure 1).

\section{2) Shanghai protocol}

Initial stimulation: Starts on day 3 of the menstrual cycle with daily CC (25 mg), plus Letrozole (2.5 mg for 4 days only). On day 6 , hMG is started every other day (150 IU), until 1 or 2 follicles have reached at least $18 \mathrm{~mm}$ in diameter. At this point a GnRH agonist (Triptorelin $100 \mathrm{mcg}$ SC) is used to trigger final oocyte maturation and OR is performed 32-36 hours later.

LPS: When the presence of at least 2 antral follicles $(2-8 \mathrm{~mm})$ is confirmed at the time of OR, follicular stimulation is re-started with daily administration of hMG (225 IU) plus Letrozole (2.5 mg) for 5-7 days. Letrozole administration stops when a dominant follicle reaches $12 \mathrm{~mm}$. In order to delay breakthrough bleeding during OR, Medroxyprogesterone (10 mg/day) is added in those cases where the leading follicle is $<14 \mathrm{~mm}$ on day 12 . Whenever there are 3 follicles with an $18 \mathrm{~mm}$ diameter or one dominant follicle exceeding $20 \mathrm{~mm}$, maturation trigger is administered in the same fashion as in phase 1, followed by OR 36 to 38 hours later (Figure 2) [10].

\section{3) Italian protocol}

Initial stimulation: Begins on day 2 of the menstrual cycle with daily doses of recombinant follicle stimulating hormone (FSHr: $300 \mathrm{IU})$ plus recombinant luteinizing hormone (LHr, 75 IU) for 4 days. Follicular tracking is started on day 5 of stimulation and performed every other day thereafter until a leading follicle reaches $13-14 \mathrm{~mm}$, at which point a $\mathrm{GnRH}$ antagonist is started until the day before ovulation. Whenever at least two follicles reach $17-18 \mathrm{~mm}$, a $\mathrm{GnRh}$ agonist is administered and OR follows 35 hours later.

LPS: 5 days after the first collection, a second stimulation begins with the same protocol used for the first stimulation and until at least two follicles reach $17-18 \mathrm{~mm}$ in diameter. Oocyte maturation is then induced with a single subcutaneous bolus of GnRH agonist, and a second OR is performed 35 hours later (Figure 3) [11].

There are different alternatives for double stimulation protocols $[7,11,12]$. These unconventional approaches in general terms are used in poor-responding patients. However, the differences are the use of GnRHa and the maximal doses accepted [4,12]. Protocols are more flexible, whilst Ubaldi et al. is rigorous [11].

Although various stimulation protocols exist and alternative approaches have been suggested, there is little consensus which technique is superior. However, an individual determination of the gonadotropin dose is strongly advised. Poorly responding patients pose a clinical challenge in assisted reproduction. Many studies have evaluated the use of various ovarian stimulation regimens to improve the outcome of poor responders undergoing IVF treatment; some of these protocols involve minimal/mild ovarian stimulation $[4,10]$.

We report four cases of women. Two of them had poor response, and the other was oncology patients aiming to preserve fertility (breast cancer and ovary borderline tumor).

\section{Case Report of Clinical Series}

These case series was conducted in New Hope Fertility Center Mexico, between March 2016 and August 2017. All participants sign the consent before inclusion in the study. Four patients were included, between 27-39 years old. Two with poor ovarian reserve (POR), based on the Bologna criteria. At least two of the following three features must be present: (I) Advanced maternal age or any other risk factor for POR; (II) A previous POR; and (III) An abnormal ovarian reserve test (ORT). Two episodes of POR after maximal stimulation are sufficient to define a patient as poor responder in the absence of advanced maternal age or abnormal ORT [6]. The other two patients had desire of fertility preservation with oncologic diagnosis. The indications and characteristics of the patients are summarized (Table 1).

To all patient transvaginal ultrasound and basal hormonal profile were done and in each one the treatments were individualized and adjusted based on the development and hormonal profile of the assessments.

First stimulation: Started with a minimal stimulation protocol. In brief, ovarian stimulation begins on early follicular phase with a daily dose of $50 \mathrm{mg}$ clomiphene citrate (CC) (Fentocile $\AA$-Ferring, Argentina) followed by human Menopausal Gonadotropin (hMG) (75 to 225 IU), (Merional ${ }^{\circledR}$ IBSA, Switzerland) injected every other day as subcutaneous injections every other day, until a leading follicle reaches 18 $\mathrm{mm}$. Trigger was instructed with 5000 iu hCG (ChoriomonIBSA ${ }^{\circledR}$, Switzerland) and OR scheduled for 36 hours later. Only follicles larger than $13 \mathrm{~mm}$ were aspirated, while smaller follicles where left untouched.

Second stimulation: With follicles $<13 \mathrm{~mm}$ on day of OR, LPS was started with daily doses of 75 iu hMG, plus $2.5 \mathrm{mg}$ Letrozole (Femara ${ }^{\circledR}$-Novartis, USA) or $50 \mathrm{mg}$ CC. After 5 days of stimulation, ultrasound and blood hormone monitoring is resumed until a leading follicle reaches $18 \mathrm{~mm}$. At this point, trigger with either human chorionic gonadotrophin (hCG) (5000 IU) or Triptorelin $50 \mu \mathrm{cg}$ (Gonapeptyl daily®-Ferring, Germany) and OR performed 36 hours later.

\section{Case 1}

A 38 years old patient, with a poor ovarian reserve in two cycles with conventional stimulation, results are in table II. This had desire of cryopreservation and pre-implantation genetic diagnosis.

Total length of stimulation for follicular and luteal phase was 10 days, with ORs performed on days 12 and 20 of cycle for follicular and luteal phase respectively. A total of 5 follicles $>14 \mathrm{~mm}$ developed during both stimulations, from which 5 MII oocytes were retrieved. All oocytes resulted in 2PNs at the fertilization check, and all reached blastocyst stage ( 2 from follicular; and 3 from luteal phase) (Table 3 ).

Once the 5 blastocysts stage confirmed, laser-assisted hatching (Hamilton-Thorne Model Zilos-tk ${ }^{\circledR}$ ) was performed on each blastocyst $(400 \mu)$, to allow for trophoectoderm cells to herniate through the zona with the help of a $30^{\circ}$ biopsy pipette (Origio, USA). Laser cell dissection followed (maximum of 3 laser triggers per biopsy) aiming to collect 5-7 cells per biopsied 


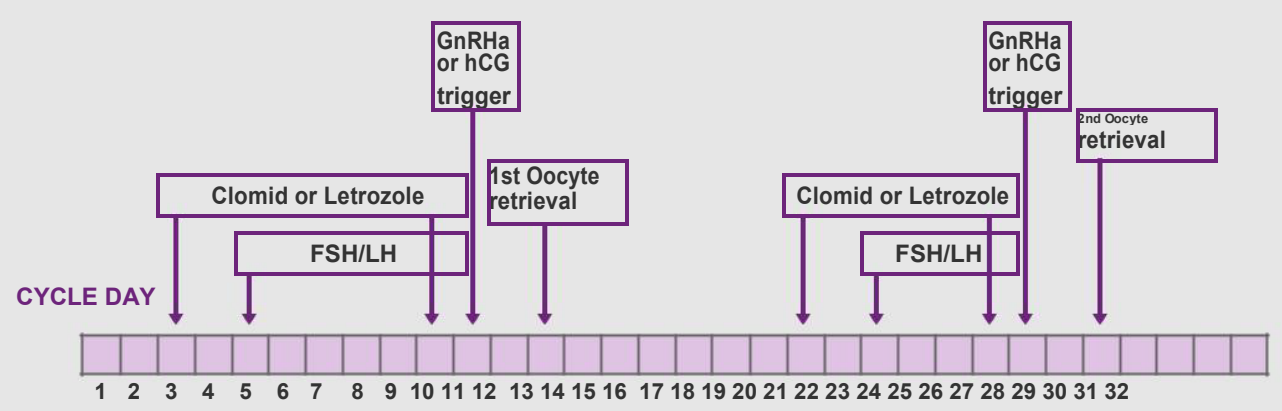

Fig. 1 - Zhang Double ovarian stimulation protocol: both follicular and luteal phase ovarian stimulation in the same cycle. GnRHa: gonadotropin releasing hormone agonist; hCG: human chorionic gonadotropin; FSH: follicle-stimulating hormone; LH: luteinizing hormone. (Zhang J, Et al.).

Figure 1: New York proposition. Zhang.

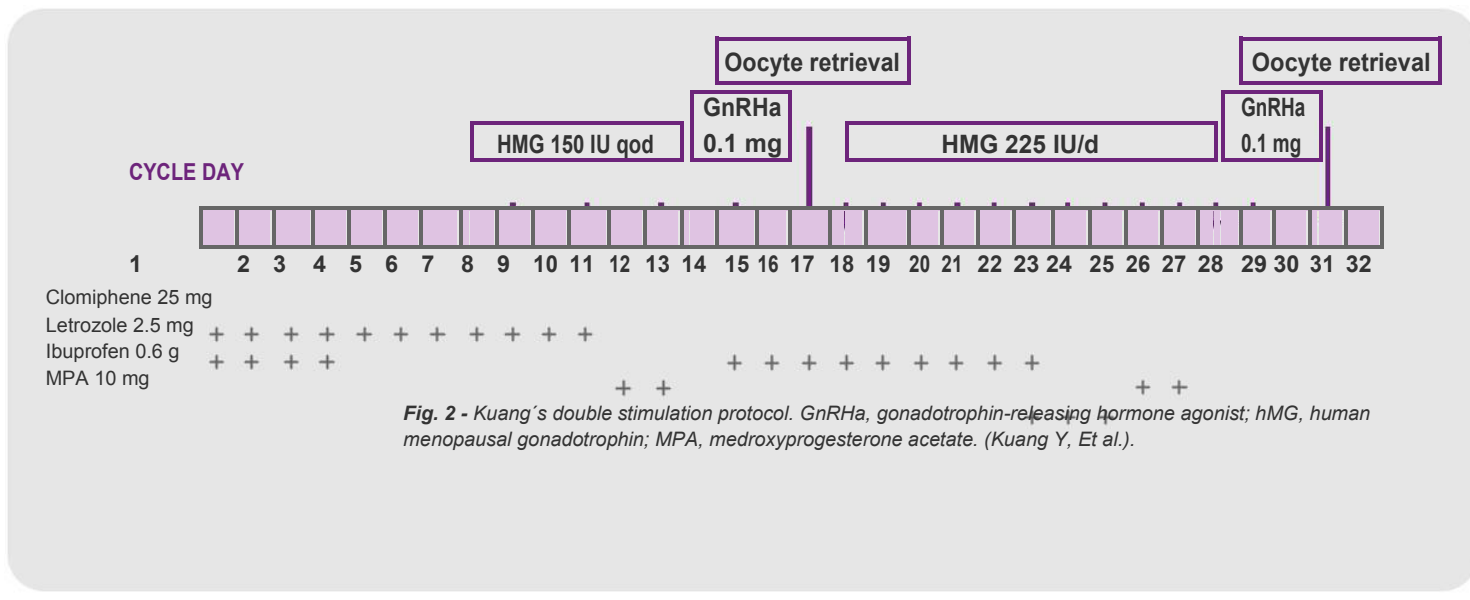

Figure 2: Shanghai protocol. Kuang et al.

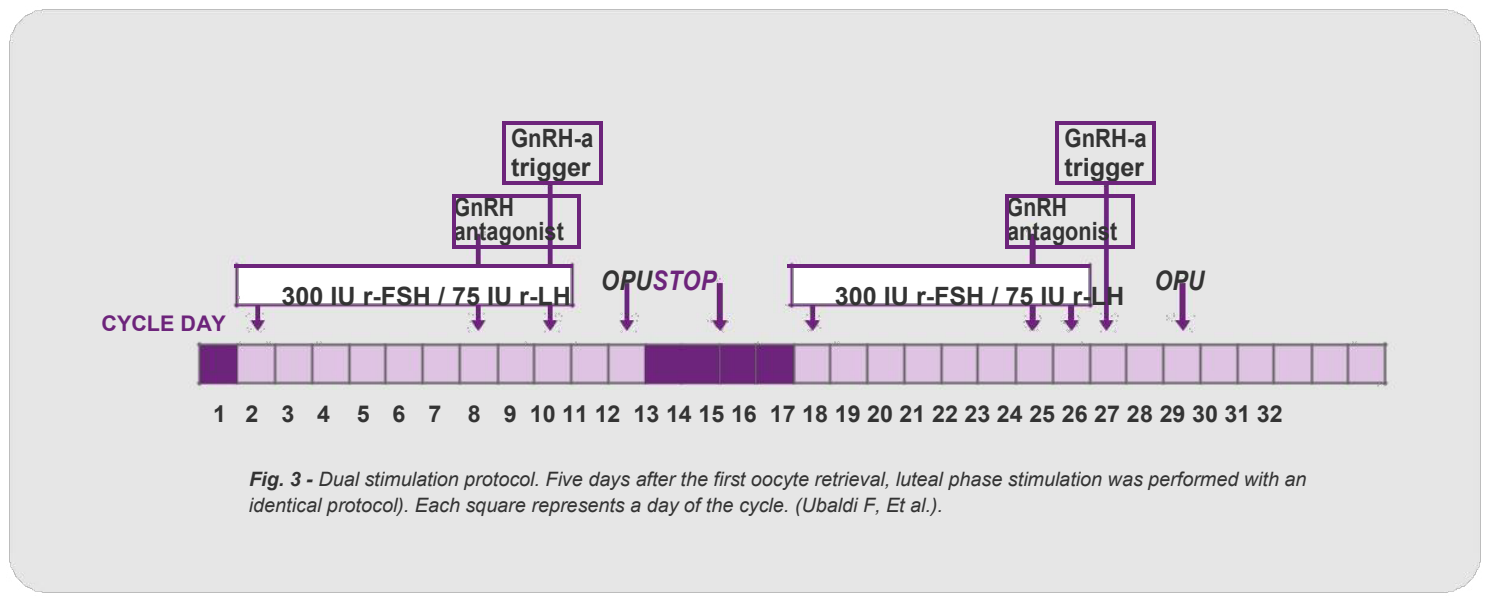

Figure 3: Italian protocol Ubaldi et al.

embryo. Retrieved cells were fixed in Eppendorf tubes with 2 $\mu 1$ PBS media (provided by Igenomix ${ }^{\circledR}$ ) and sent out at $-20^{\circ} \mathrm{C}$ for PGT-A with NGS (Igenomix ${ }^{\circledR}$ Mexico). Biopsied embryos were cryopreserved with the Cryotop method (Kitazato, Biopharma, Japan). Kuwayama (2007) from these, 2 blastocysts were reported as $46 \mathrm{XX}$ (euploid), one from each stimulation phase as we can see (Table 4). We summarized both phases of stimulation and described days of stimulation, oocyte MII, oocyte fertilized, biopsied, euploids and total dose of hMG (Table 5).

\section{Case 2}

40 years olds patient, with a history of 1 miniFIV ${ }^{\circledR}$ canceled by the request of the couple. The average of the total length of stimulation for follicular and luteal phase was 12 days. Seven follicles $>14 \mathrm{~mm}$ developed during both stimulations from which seven MII oocytes were retrieved. Five oocytes resulted in 2PNs at the fertilization check and just one embryo reached cleavage stage (from luteal phase stimulation) (Table 6 and 7$)$. 
Citation: Olmedo LMC, Rioja MDJL, González CMS, et al. Lutheal phase stimulation and fertility and first outcomes. Gynecol Reproduct Endocrinol-UK. 2018;2(3): 5-10

Table 1: Baseline characteristics of the patient indication and history of TRA.

\begin{tabular}{|c|c|c|c|}
\hline Case & Age (year) & Indication & Previous treatment \\
\hline 1 & 38 & Poor ovarian reserve & $\begin{array}{c}2 \text { previous cycles of conventional } \\
\text { stimulation }\end{array}$ \\
\hline 2 & 38 & Poor ovarian reserve & $\begin{array}{c}2 \text { previous cycles with miniFIV } \\
\text { stimulation }\end{array}$ \\
\hline 3 & 39 & Breast cancer & Without reproductive treatment \\
\hline 4 & 27 & $\begin{array}{l}\text { Ovary borderline } \\
\text { tumor }\end{array}$ & Without reproductive treatment \\
\hline
\end{tabular}

Table 2: Results from previous cycles.

\begin{tabular}{|c|c|c|c|c|}
\hline Cycle number & $\begin{array}{c}\text { Oocytes } \\
\text { retrieved }\end{array}$ & $\begin{array}{c}\text { Oocyte } \\
\text { fertilized }\end{array}$ & $\begin{array}{c}\text { Blasts } \\
\text { obtained }\end{array}$ & $\begin{array}{c}\text { Euploid } \\
\text { embryos }\end{array}$ \\
\hline $1^{\circ}$ Follicular & 2 & 2 & 2 & 0 \\
\hline $2^{\circ}$ Follicular & 4 & 4 & 2 & 0 \\
\hline
\end{tabular}

Table 3: Results from follicular and luteal stimulation per cycle.

\begin{tabular}{|c|c|c|c|}
\hline Cycle number & $\begin{array}{c}\text { Oocytes } \\
\text { retrieved }\end{array}$ & Oocyte fertilized & Blasts obtained \\
\hline $3^{\circ}$ Follicular & 2 & 2 & 2 \\
\hline $3^{\circ}$ Luteal & 3 & 3 & 3 \\
\hline
\end{tabular}

Table 4: Trophoectoderm biopsy result of all the blastocysts.

\begin{tabular}{|c|c|c|c|}
\hline Stimulation & PGT-A & PGT-A Results & hGC \\
\hline $3^{\circ}$ Follicular & NGS & Abnormal & \\
\hline $3^{\circ}$ Follicular & NGS & Normal & \\
\hline $3^{\circ}$ Luteal & NGS & Abnormal & \\
\hline $3^{\circ}$ Luteal & NGS & Abnormal & \\
\hline $3^{\circ}$ Luteal & NGS & Normal & Positive \\
\hline
\end{tabular}

Table 5: Summary of the results from follicular and luteal stimulation.

\begin{tabular}{|c|c|c|}
\hline & Follicular & Luteal \\
\hline Days of stimulation & 7 & 3 \\
\hline Oocyte in MII & 2 & 3 \\
\hline Fertilized oocyte & 2 & 3 \\
\hline Biopsed & 2 & 3 \\
\hline Euploid blastocysts & 1 & 1 \\
\hline Total dose of hMG & $825 \mathrm{IU}$ & $225 \mathrm{IU}$ \\
\hline
\end{tabular}

\section{Case 3}

A 39 years olds patient, had desire of fertility preservation for oncologic diagnosis (Breast cancer). This began an emergency stimulation in luteal phase at day 19. A total length of stimulation for luteal phase and follicular was 23 days and 16 respectively. A total of 10 follicles $>14 \mathrm{~mm}$ developed during both stimulations from which 10 MII oocytes were retrieved, 6 oocytes resulted in 2PNs at the fertilization check and all 6 reached cleavage stage (5 from follicular; and 1 from luteal phase) (Table 8 and 9).

\section{Case 4}

A 27 years olds patient had desire of fertility preservation for oncologic diagnosis (Ovary Borderline tumor). This began stimulation in luteal phase (day 17). A total length of stimulation in luteal phase was 7 days, with ORs performed on days 25 for LPS. A total of 8 follicles $>14 \mathrm{~mm}$ developed during both stimulations from which 8 MII oocytes were retrieved. Seven oocytes resulted in 2PNs at the fertilization check and three reached D2, D3 and 2 blastocyst stage (Table 10 and 11).

\section{Discussions}

Innovations in stimulation regimens have been an integral
Table 6: Results from follicular and luteal stimulation.

\begin{tabular}{|c|c|c|c|c|}
\hline Cycle number & $\begin{array}{c}\text { Oocytes } \\
\text { retrieved }\end{array}$ & $\begin{array}{c}\text { Oocytes } \\
\text { fertilized }\end{array}$ & Embryo day 3 & $\begin{array}{c}\text { Blasts } \\
\text { obtained }\end{array}$ \\
\hline $1^{\circ}$ Follicular & Cancel & - & - & - \\
\hline $2^{\circ}$ Follicular & 1 & 1 & 0 & - \\
\hline $2^{\circ}$ Luteal & 1 & 0 & - & - \\
\hline $3^{\circ}$ Follicular & 1 & 1 & 0 & - \\
\hline $3^{\circ}$ Luteal & 1 & 1 & 0 & - \\
\hline $4^{\circ}$ Follicular & 2 & 1 & 0 & - \\
\hline $4^{\circ}$ Luteal & 1 & 1 & 1 & - \\
\hline
\end{tabular}

Table 7: Summary of the results from follicular and luteal stimulation.

\begin{tabular}{|c|c|c|}
\hline & Follicular & Luteal \\
\hline Days of stimulation & 7 & 5 \\
\hline Oocyte in MII & 4 & 3 \\
\hline Fertilized oocytes & 3 & 2 \\
\hline Embryo day 3 & 0 & 1 \\
\hline Blastocysts & 0 & 0 \\
\hline Total dose of hMG & $375 \mathrm{UI}$ & $375 \mathrm{UI}$ \\
\hline
\end{tabular}

Table 8: Results from follicular and luteal stimulation.

\begin{tabular}{|c|c|c|c|c|}
\hline Cycle number & $\begin{array}{c}\text { Oocytes } \\
\text { retrieved }\end{array}$ & $\begin{array}{c}\text { Oocytes } \\
\text { fertilized }\end{array}$ & Day 3 & $\begin{array}{c}\text { Blasts } \\
\text { obtained }\end{array}$ \\
\hline $1^{\circ}$ Luteal & 4 & 1 & 1 & 0 \\
\hline $2^{\circ}$ Follicular & 6 & 5 & 5 & 0 \\
\hline
\end{tabular}

Table 9: Summary of the results from follicular and luteal stimulation.

\begin{tabular}{|c|c|c|}
\hline & Follicular & Luteal \\
\hline Days of stimulation & 14 & 9 \\
\hline Oocyte in MII & 4 & 6 \\
\hline Fertilized oocytes & 1 & 5 \\
\hline Cleavage stage & 1 & 5 \\
\hline Blastocysts & 0 & 0 \\
\hline Total dose of hMG & $1500 \mathrm{UI}$ & $1125 \mathrm{UI}$ \\
\hline
\end{tabular}

Table 10: Trophoectoderm biopsy result of all blastocysts.

\begin{tabular}{|c|c|c|c|c|}
\hline Cycle number & $\begin{array}{c}\text { Oocytes } \\
\text { retrieved }\end{array}$ & $\begin{array}{c}\text { Oocytes } \\
\text { fertilized }\end{array}$ & $\begin{array}{c}\text { Blasts } \\
\text { obtained }\end{array}$ & $\begin{array}{c}\text { Euploid } \\
\text { embryos }\end{array}$ \\
\hline $1^{\circ}$ Luteal & 8 & 7 & 2 & 0 \\
\hline
\end{tabular}

Table 11: Results from luteal stimulation.

\begin{tabular}{|c|c|}
\hline & Luteal \\
\hline Days of stimulation & 7 \\
\hline Oocyte in MII & 8 \\
\hline Fertilized oocytes & 7 \\
\hline Embryo day 2 & 3 \\
\hline Embryo day 3 & 2 \\
\hline Blastocysts & 2 \\
\hline Total dose of FSH & 975 \\
\hline
\end{tabular}

part of IVF treatment for the past 30 years. Poor response is estimated to happen on 9 to $24 \%$ of all stimulated cycles [4] and although several stimulation protocols have been described in order to maximize oocyte yield per started cycle, most fail to successfully attain their goal despite modifications on type or amount of gonadotrophins selected, use of GnRH agonist priming or even following the addition of adjuvants to stimulation such as the use of growth hormone or DHEA, amongst others $[11,13]$. However, recent advances on what we know about folliculogenesis, particularly the concept of two or more cohorts of antral follicles happening during each cycle $[4,12,14]$, have become the base on which unconventional stimulation protocols have developed (luteal phase stimulation 
and double stimulation protocols included), allowing for a second opportunity to retrieve good quality oocytes within a cycle, conferring advantages which could prove to be critical under certain circumstances.

Patients with oncologic diagnosis, poor ovarian reserve, or even patients with a poor ovarian response, during follicular stimulation despite having a good ovarian reserve (e.g. PCOS patients) could benefit from unconventional protocols. In particular double stimulation protocols, could give the advantage of avoiding the emotional distress of having to wait for another cycle and to face uncertainty of a possible adverse outcome.

Several double stimulation protocols previously described to improve the number of egg cells and embryos per cycle. The mild ovarian-stimulation approach administers either lower doses (over fewer days) of exogenous gonadotropins, or other compounds (such as antiestrogens, aromatase inhibitors, or GnRH-antagonist) $[9,15]$. This regimen can reduce patient discomfort and risk, and lower the duration and intensity of the pharmacological interventions $[9,15]$. On the other hand second phase (luteal phase) stimulation as a sequence of a mild stimulation can be started in the following days after an egg retrieval in cases in which the ovarian response is compromised or low, thus helping to alleviate the patients psychological distress [16], reported the egg retrieval rate was higher in the first luteal phase stage. However, there were no significant differences in the mature egg cell percentage obtained at each stimulation stage, fertilization rate and cryopreservation. There were no significant differences between the first and second collection in terms of embryo quality or the number of cryopreserved embryos. The implantation rate was similar in the embryos obtained from first phase stimulation and second phase $[4,11,15]$ had similar findings in the number of oocytes, fertilization rate, blastocyst stage embryos and finally euploid embryo rate in both stimulation phases. Thus these authors consider this protocol as an alternative for low ovarian reserve allow offering patients a higher rate of oocytes and potentially viable embryos within a shorter timeframe.

We selected Zhang protocol for these series of cases from the others for its flexibility and because it was easily adapted to our case. With a lower amount of medication and more friendly for patients with limited response, higher stress levels and limited budget. Although the Kuang et al. $[4,16]$ protocol offers a good alternative for its similarity to Zhang protocol in the timeframe, groups of medications employed and flexibility, however it was not an option in this specific case since similar to the Italian protocol, both use high stimulation doses which had previously proved to be ineffective in this specific patient.

The current tendency in reproductive technologies and embryo selection is to limit the invasiveness, costs and risks. The current and safest approach for embryo selection is the trophoectoderm biopsy and PGT-A, since it has proven less potential damage to the embryos and more available DNA samples $[17,18]$. This technique combined with new technologies for genetic testing such as NGS offer faster, more precise and sensitive results for embryo euploidy, at a fraction of the cost in comparison to previous technologies (eg. aCGH) [18,19]. Having considered our patient's age, her reduced ovarian response, the advanced maternal age and couple's concern for the potential aneuploidies we recommended in this particular case the application of PGT-A with NGS. PGT-A not only improves the pregnancy rate per embryo transfer, it also reduce the pregnancy loss rate related to aneuploidy and its implications with reduced ovarian reserve couples $[18,20]$.

To the best of our knowledge, this is the first report of a series of cases in Mexico and Latin America where the luteal phase and pre-implantation genetic screening are in combination through NGS. The hallmark of this case is that this patient was enabled to achieve two euploid embryos during a single cycle, despite an initial low number of oocytes retrieved due to the second stimulation in the luteal. However, the number of biopsied embryos was higher during the luteal phase increasing the chances of achieving a euploid embryo and potentially a live birth. This outcome was not reached during the two previous cycles that the patient underwent and this technique improved the total number of oocytes and blastocyst obtained during a single cycle divided in two stages. In addition, the amount of euploid embryos was the same, which is consistent with the current reports from other authors.

Patients presenting with challenging stimulations, such as the case, should not only be offered conventional stimulation protocols. As clinicians, we should aim to pursue optimal personalized stimulations for each of these patients, incorporating new protocols, which in turn will allow us to providing more flexibility, and potentially better outcomes. In our view, double stimulation protocols seem to be effective strategies in the poor responders and where time is limited to one cycle.

\section{Conclusions}

The efficiency of the luteal-phase stimulation protocols is promising. Nowadays, it is a useful technique for lowresponders to increase the final number of oocytes and potential embryos in comparison to single stimulation protocols Zhang and Ubaldi et al. [4,11,15]. However, current indications for double stimulation protocols shouldn't be limited to low responders since it could become an alternative which could be offered to patients with spontaneous ovulation, in cases of emergency cryopreservation in oncology patients and women with time constrains for whom shortening times towards an egg retrieval is desirable.

\section{Funding}

The present study was supported by New Hope Fertility Center, Ciudad de México

\section{Acknowledgement}

The authors thank the staff of New Hope Fertility Center, Ciudad de México.

\section{References}

1. Baerwald A, Adams G, Pierson R. Ovarian antral folliculogenesis during the human menstrual cycle: A review. Hum Reprod Update. 2012;18(1):73-91.

2. Bentov Y, Esfandiari N, Gokturk A, et al. An ongoing pregnancy from two waves of follicles developing during 
a long follicular phase of the same cycle. Fertil Steril. 2010;94(1):350.e8-11.

3. Lueders I, Niemuller C, Hildebrandt. Gestating for 22 months: Luteal development and pregnancy maintenance in elephants. Proc Biol Sci. 2012;279(1743):3687-96.

4. Zhang J. Luteal phase ovarian stimulation following oocyte retrieval: Is it helpful for poor responders? Reprod Biol Endocrinol. 2015;13:76-80.

5. Buendgen N, Schultze A, Cordes T, et al. Initiation of ovarian stimulation independent of the menstrual cycle: A case-control study. Arch Gynecol Obstet. 2013;288(4):901-4.

6. Maman E, Meirow D, Brengauz M, et al. Luteal phase oocyte retrieval and in vitro maturation is an optional procedure for urgent fertility preservation. Fertil Steril. 2011;95(1):64-7.

7. Cakmak H, Katz A, Cedars MI, et al. Effective method for emergency fertility preservation: Random-start controlled ovarian stimulation. Fertil Steril. 2013;100(6):1673-80.

8. Moffat R, Pirtea P, Gayet V, et al. Dual ovarian stimulation is a new viable option for enhancing the oocyte yield when the time for assisted reproductive technology is limited. Reprod Biomed Online. 2014;29(6):659-61.

9. Fauser B, Nargund G, Andersen A, et al. Mild ovarian stimulation for IVF: 10 years later. Hum Reprod. 2010;25(11):2678-84.

10. Kuang Y, Chen Q, Hong Q, et al. Double stimulations during the follicular and luteal phases of poor responders in IVF/ICSI programmes (Shanghai protocol). Reprod Biomed Online. 2014;29(6):684-91.

11. Ubaldi F, Capalbo A, Vaiarelli A, et al. Follicular versus luteal phase ovarian stimulation during the same menstrual cycle (DuoStim) in a reduced ovarian reserve population results in a similar euploid blastocyst formation rate: New insight in ovarian reserve exploitation. Fertil Steril. 2016;105(6):1488-95.

12. Kuang Y, Hong Q, Chen Q, et al. Luteal phase ovarian stimulation is feasible for producing competent oocytes in women undergoing in vitro fertilization/intracytoplasmic sperm injection treatment, with optimal pregnancy outcomes in frozen thawed embryo transfer cycles. Fertil Steril. 2014;101(1):105-11.

13. Sunkara SK, Coomarasamy A. Androgen pretreatment in poor responders undergoing controlled ovarian stimulation and in vitro fertilization treatment. Fertil Steril. 2011;95(8):e73-5.

14. Zegers-Hochschild F, Adamson G, Mounzon J, et al. The International Committee for Monitoring Assisted Reproductive Technology (ICMART) and the World Health Organization (WHO) revised glossary on ART terminology, 2009. Hum Reprod. 2009;24(11):2683-87.

15. Ubaldi F, Vaiarelli A, D'Anna R, et al. Management of poor responders in IVF: Is there anything new? BioMed Res Inter. 2014:1-10.

16. Kuang Y, Quiuju C, Qinquing H, et al. Luteal phase ovarian stimulation case report: Three-year follow-up of a twin birth. J IVF Reprod Med Genet. 2013;1(2):1-3.

17. Yang Z, Lin J, Zhang J, et al. Randomized comparison of next-generation sequencing and array comparative genomic hybridization for pre-implantation genetic screening: a pilot study. BMC Med Genomics. 2015;8:30.

18. Yang D, Yang W, Li Y, et al. Progress in understanding human ovarian folliculogenesis and its implications in assisted reproduction. J Assist Reprod Genet. 2013;30(2):213-9.

19. Ferraretti AP, La Marca A, Fauser BC, et al. ESHRE consensus on the definition of 'poor response' to ovarian stimulation for in vitro fertilization: The bologna criteria. Hum Reprod. 2011;26(7):1616-24.

20. Hee J, Henderson S, Garcia E. Successful live birth after transfer of blastocyst and frozen blastocyst from rescue ICSI with application of polarized light microscopy for spindle examination on unfertilized eggs. J Ovarian Res. 2015;8:22.

\section{*Correspondence to:}

Luis M Campos Olmedo

Medical Coordinator

New Hope Fertility Center

Mexico Rome

Tel: +5215551449073

E-mail: drluismiguel-campos@nhfc.mx 